\title{
Organization of the Escherichia coli and Salmonella typhimurium chromosomes between flagellar regions IIIa and IIIb, including a large non-coding region
}

\author{
Manidipa Raha, May Kihara, Ikuro Kawagishi $\dagger$ and Robert M. Macnab* \\ Department of Molecular Biophysics and Biochemistry, Yale University, New Haven, Connecticut 06511, USA
}

(Received 15 March 1993; revised 15 April 1993; accepted 26 April 1993)

\begin{abstract}
Flagellar regions IIIa and IIIb of the Escherichia coli and Salmonella typhimurium chromosomes (at 40 min and 42-43 min, respectively) has been shown to be separated by DNA unrelated to flagellar function, with region IIIa being immediately followed by a gene, amy $A$, that encodes a cytoplasmic $\alpha$-amylase. The chromosome between amy $A$ and flagellar region IIIb has now been investigated. The high level of DNA similarity between the $E$. coli and $S$. typhimurium sequences that exists in flagellar region IIIa and in amy $A$ continues initially, with three genes of unknown function; in $E$. coli, there may be a fourth gene. The remainder of the region, up to the start of flagellar region IIIb, lacks any obvious open reading frames, scores poorly on an algorithm for coding probability, has a high $\mathrm{A}+\mathrm{T}$ content, and is totally dissimilar in the two species. We conclude that it is non-coding. In $E$. coli this region extends for $2.7 \mathrm{~kb}$ and in $S$. typhimurium for $0.8 \mathrm{~kb}$. These values are unusually large for prokaryotes, where the non-coding regions between operons are generally quite short. The data, which are discussed in the context of a hypothesized disruption of a contiguous ancestral flagellar region, may give new insight into the organization and evolution of the bacterial chromosome.
\end{abstract}

\section{Introduction}

In contrast to eukaryotic genomes, bacterial genomes are compact, with typically only around $50-300$ bp of noncoding sequence between adjacent operons. For example, in the flagellar regulons of Salmonella typhimurium and Escherichia coli, (subscripts $\mathrm{E}$ and $\mathrm{S}$ are used throughout this paper to distinguish $E$. coli and $S$. typhimurium genes) among the cases where the non-coding distance between adjacent operons has been established, the mean value is $151 \mathrm{bp}$, the highest value is $263 \mathrm{bp}$ (between the $f i C_{\mathrm{E}}$ and $f i D_{\mathrm{E}}$ operons of $E$. coli; Hanafusa et al., 1989), and the lowest value is $64 \mathrm{bp}$ (between the $f g B_{\mathrm{S}}$ and $f g K_{\mathrm{S}}$ operons of S. typhimurium; Homma et al., 1990).

We showed recently (Kawagishi et al., 1992) that a set of 19 flagellar genes in $E$. coli, which originally had been called flagellar region III, in fact consists of two contiguous sets (region IIIa, containing 5 genes, and

* Author for correspondence. Tel. (203) 432 5590; fax (203) 432 5592; e-mail rmacnab@yalevm.ycc.yale.edu.

$\dagger$ Present address: Department of Molecular Biology, Nagoya University, Nagoya, Japan.

The nucleotide sequence data reported in this paper have been submitted to GenBank and have been assigned the accession numbers L13279 and L13280. region IIIb, containing 14 genes) separated by about $6.6 \mathrm{~kb}$; a similar situation exists in S. typhimurium except that the intervening region is smaller $(4.4 \mathrm{~kb})$. Thus the last operon in flagellar region IIIa and the first operon in flagellar region IIIb are separated by a large distance. What is the nature and function of this DNA? Chromosomal deletion established that it contains no genes necessary for motility or for normal growth rates on minimal medium, but of course this does not exclude the possibility that it contains non-essential genes. Indeed, this intervening region is so large that it would be surprising if it was entirely non-coding.

The supposition that it would contain at least some coding sequence was soon vindicated by the finding that the last flagellar operon in region IIIa (the $f i D$ operon) was immediately followed by a gene, amy $A$, which encodes a cytoplasmic $\alpha$-amylase (Raha et al., 1992). We have now examined the entire intervening region, and conclude that although it does contain several genes, it also contains an unusually long stretch of non-coding sequence.

\section{Methods}

Bacterial strains. E. coli strains DH5 $\alpha$ (Gibco BRL) and XL1-Blue and JM101 (Stratagene) were used for cloning, subcloning and M13 production. 
Cloning and sequencing of E. coli DNA. pIK1001 is a pBR322-based plasmid with an insert that contains the $f i D_{\mathrm{E}}$ operon of flagellar region IIIa, the entire intervening region, and flagellar region IIIb genes from $f i E_{\mathrm{E}}$ through $f i F_{\mathrm{E}}$ to an $E c o$ RI site in $f i G_{\mathrm{E}}$ (Kawagishi et al., 1992). A $2 \cdot 1 \mathrm{~kb}$ fragment of the insert between the MluI and $X b a \mathrm{I}$ sites was subcloned into pIBI25 (International Biotechnologies Inc.) to give plasmid pMR7. Using the $X b a I$ site and the vector HindIII site, the cloned fragment from pMR7 was recloned into the corresponding sites in plasmid Bluescript KS (Stratagene) to give plasmid pMR8.

The approaches used to sequence $a m y A_{\mathrm{E}}$, and the DNA up to a $M l u \mathrm{I}$ site $2.2 \mathrm{~kb}$ beyond $a m y A_{\mathrm{E}}$ have been described (Raha et al., 1992), as have those for the DNA from an $X b a \mathrm{I}$ site $0.7 \mathrm{~kb}$ before $f i E_{\mathrm{E}}$ through that gene (Müller et al., 1992). Exonuclease III deletions were generated from both ends of the inserts of pMR7 and pMR8, using the $X b a \mathrm{I}$ and $K p n I$ sites of pMR7 and the HindIII and KpnI sites of pMR8; these were then used for sequencing by the dideoxy chain termination method using commercial synthetic primers. Both the $M l u \mathrm{I}$ and the $X b a I$ sites were sequenced through, using appropriate plasmids.

Cloning and sequencing of S. typhimurium DNA. pOYA1 is a pUC119based plasmid (Müller et al., 1992); it has a $6.7 \mathrm{~kb} E c o$ RI-EcoRI insert of $S$. typhimurium DNA that extends from within $f i D_{\mathrm{S}}$ in flagellar region IIIa through all of the intervening region and as far as $f i F_{\mathrm{S}}$ in flagellar region IIIb.

The approaches used to sequence $a m y A_{\mathrm{S}}$ and $f i E_{\mathrm{S}}$ have been described (Raha et al., 1992; Müller et al., 1992). For sequencing the rest of the intervening region, four M13 derivatives of pOYA1 were used. The first two contained a ClaI-HpaI fragment (from within $a m y A_{\mathrm{S}}$ to near the end of orf $48_{\mathrm{S}}$ ) cloned into M13mp18 and M13mp19, while the second two contained a BamHI-EcoRI fragment (from within orf $48_{\mathrm{S}}$ to within $f i F_{\mathrm{S}}$ ) cloned into the same vectors.

Chemicals and enzymes. All chemicals and enzymes were obtained from standard commercial sources. Antibiotics were used at the following concentrations: ampicillin, $50 \mu \mathrm{g} \mathrm{ml}^{-1}$; tetracycline, $10 \mu \mathrm{g} \mathrm{ml}^{-1}$. Synthetic primers (18-mers) were synthesized using an Applied Biosystems 392 DNA/RNA Synthesizer.

\section{Results}

Overall organization of the intervening regions in E. coli and $S$. typhimurium

The $f i D_{\mathrm{E}}$ operon in flagellar region IIIa is followed by an amylase gene, $a m y A_{\mathrm{E}}$, oriented clockwise on the chromosome (Raha et al., 1992). We have now extended the sequence analysis all the way through the intervening region to the $3^{\prime}$ end of $f i E_{\mathrm{E}}$ (see Methods for details). The results are shown schematically in Fig. 1(a); the corresponding DNA sequence is available in GenBank under accession number L13279.

The fragment used for analysing $a m y A_{\mathrm{E}}$ contained a second open reading frame oriented counterclockwise, with a short $(77 \mathrm{bp})$ distance between the $3^{\prime}$ ends of the respective coding regions. This second open reading frame has a deduced product molecular mass of $15 \mathrm{kDa}$, and will be referred to as $\operatorname{orf} 15_{\mathrm{E}}$. Diverging from the $5^{\prime}$ non-coding region of orf15 $\mathrm{E}$ were two open reading frames with an ATGA overlap between them; both had strong consensus ribosome binding sequences. They correspond to products with deduced molecular masses of 48 and $9 \mathrm{kDa}$, respectively, and so will be referred to as $\operatorname{orf} 48_{\mathrm{E}}$ and $\operatorname{orf} 9_{\mathrm{E}}$. About $100 \mathrm{bp}$ following the $3^{\prime}$ end of $\operatorname{orf}_{\mathrm{E}}$ was a fourth short open reading frame, $\operatorname{orf}_{\mathrm{E}}$, with an extremely strong, well-placed consensus ribosome binding sequence (AGGAGG), and followed by a pronounced stem-loop sequence. Based on their opposite orientation, $\operatorname{amy} A_{\mathrm{E}}, \operatorname{orf} 15_{\mathrm{E}}$ and $\operatorname{orf} 48_{\mathrm{E}}$ must belong to distinct transcriptional units. With a $4 \mathrm{bp}$ overlap of their coding regions, orf $48_{\mathrm{E}}$ and $\operatorname{orf}_{\mathrm{E}} \mathrm{E}_{\mathrm{E}}$ presumably belong to the same transcriptional unit, while the distance between the $3^{\prime}$ end of $\operatorname{orf}_{\mathrm{E}}$ and the $5^{\prime}$ end of $\operatorname{orf}_{\mathrm{E}}$ suggests that the latter is a separate transcriptional unit. It is not obvious from inspection what the corresponding promoters are and, in the absence of knowledge regarding gene product function (see below), we did not attempt to determine the transcription start sites.

For the next $2.7 \mathrm{~kb}$, until the first flagellar gene in region $\mathrm{IIIb}, f i E_{\mathrm{E}}$, there was no evidence for any further genes: open reading frames in both orientations were very short (mostly less than $300 \mathrm{bp}$ ) and had no obvious ribosome binding site.

The intervening region of $S$. typhimurium followed a similar pattern (Fig. $1(b)$; sequence available in GenBank under accession number L13280), with two exceptions: there was no evidence for an open reading frame corresponding to $\operatorname{orf} 8_{\mathrm{E}}$, and the distance between the last open reading frame, $\operatorname{orf}_{\mathrm{S}}$, and $f i E_{\mathrm{S}}$ was much shorter $(0.8 \mathrm{~kb})$ than in E. coli.

\section{Comparison of the DNA sequence of the intervening region in the two species}

When DNA sequences of the intervening region in $E$. coli and $S$. typhimurium were compared in a matrix plot (Fig. 2 ), the similarity seen previously in flagellar region IIIa and amy $A$ was found to extend through orf15, orf 48 and orf 9 . These open reading frames in the two species show 77,81 and $86 \%$ identity, respectively, when aligned by the algorithm of Lipman \& Pearson (1985).

Following orf 9 , no sequence similarity was discerned, either by inspection of Fig. 2 or by attempted alignment, until the start of flagellar region IIIb. Thus orf $_{\mathrm{E}}$ plus the $2.7 \mathrm{~kb}$ between $\operatorname{orf}_{\mathrm{E}}$ and $f i E_{\mathrm{E}}$ is unrelated to the $0.8 \mathrm{~kb}$ between orf9 $9_{\mathrm{S}}$ and $f l E_{\mathrm{S}}$. If they were part of the same sequence originally, they must since have diverged beyond recognition, and also (at least in the case of $S$. typhimurium) undergone further rearrangement by deletion.

\section{Evidence that orf15, orf48 and orf9 are authentic genes}

Minicell analysis (Raha et al., 1992) has demonstrated that orf15 encodes a $15 \mathrm{kDa}$ protein and so is an authentic gene. We conclude that orf 48 and orf 9 are 
(a) E. coli

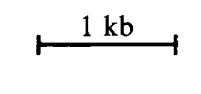

Flagellar region JIIa

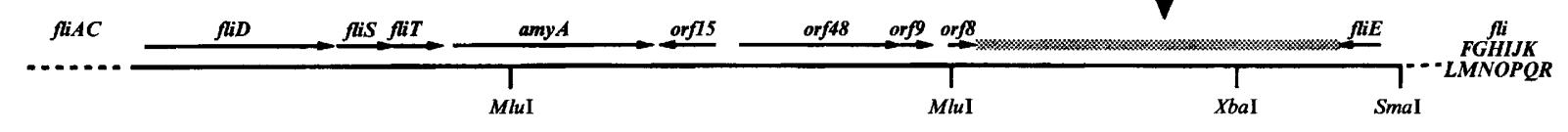

(b) S. typhimurium

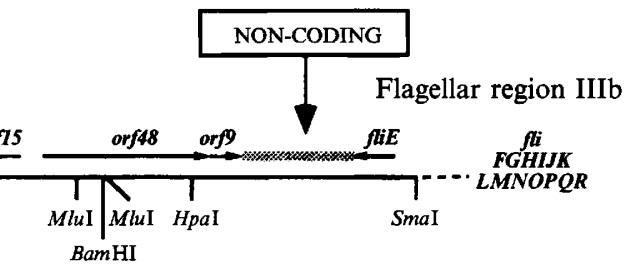

Fig. 1. The chromosomes of (a) E. coli and (b) S. typhimurium in the vicinity of flagellar regions IIIa and IIIb. Region IIIa contains $f i A, f i B$ (S. typhimurium only), fliC, and the $f i D$ operon, consisting of $f i D, f i S$ and $f i T$. Region IIIb contains $f i E$, and the $f i F G H I J K$ and fiLMNOPQR operons. In the intervening region following the fiD operon are amyA (encoding an $\alpha$-amylase), orf 15 , orf 48 , orf 9 , and (in E. coli) orf 8 ; arrows indicate the extent of these open reading frames. With a 4 bp overlap in their coding regions, orf 48 and orf9 are presumably in the same operon; orf15 and orf8 (E. coli) appear to be single-gene transcriptional units. The rest of the intervening region (stippled) is non-coding (see text) and is unusually long for a prokaryote. It shows no sequence similarity between $E$. coli and $S$. typhimurium and differs considerably in length $(2.7 \mathrm{~kb}$ vs. $0.8 \mathrm{~kb})$.

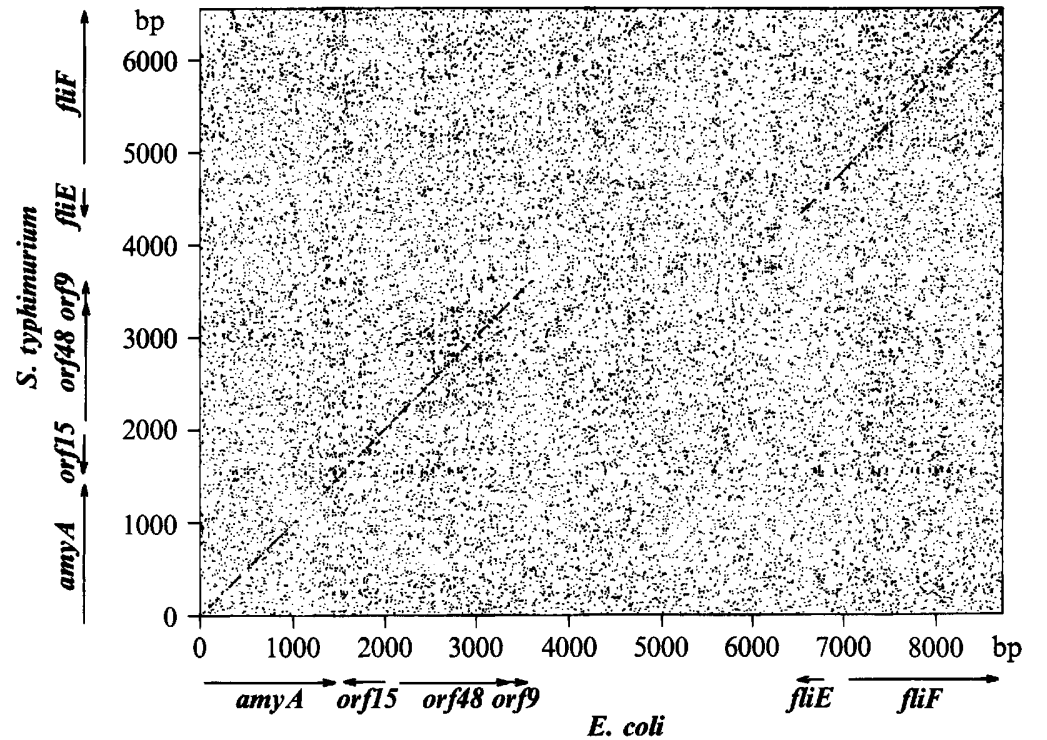

Fig. 2. Similarity matrix of the $S$. typhimurium and $E$. coli chromosomes at around $42-43$ and 40 min on their respective maps. The comparison starts with the amylase gene amyA (the first following flagellar region IIIa) and continues through the early part of flagellar region IIIb. From amy $A$ through orf 9 , and again from $f i E$ on, the sequences are very similar, whereas between $\operatorname{orf} 9$ and $f i E$ there is no detectable similarity. The sequences were scanned on a window of six bases. authentic genes also, based on the following considerations.

The strongest evidence is that the deduced amino acid sequences show even higher levels of identity than the corresponding nucleotide sequences $(83,92$ and $100 \%$ for Orf15, Orf48 and Orf9, respectively; Fig. 3). This result is statistically extremely improbable for noncoding DNA, because of the much larger basis set of 20 amino acids vs. that of four nucleotides. Inspection of the genetic code reveals that a one-base codon change has a probability of 0.76 of causing an amino acid change, and so between closely related sequences (where changes of more than one base per codon can be neglected) an $x \%$ difference in nucleotide sequence (corresponding to a $3 x \%$ difference in codons) will statistically yield an amino acid difference of $2 \cdot 3 x \%$. [In the more general case where multiple changes per codon cannot be neglected, computer simulation demonstrates that translation of two DNA sequences which differ from each other by random changes to a given extent (say, $20 \%$ ) generates deduced amino acid sequences that are much more different (in this case about 40\%).] As expected from the genetic code, the nucleotide alignments for $\operatorname{orf15}$, orf 48 and $\operatorname{orf} 9$ show that mismatches at the 
(a) Orf15 (83\% identity)

Ec 1 MKKLAIAGAL LLLAGCAEVE NYNNVUKTPA PDWLAGYWT KGPQRALYSP EAIGSLIVTK EGDTLDCRQW QRVIAVPGKL TLMSDDLTNY TVKRELYEVE

St 1 MKKVAIVGAL LVLAGCAEVE NYWDVVKTPA PAGLEGYOS KGPQRKLYSP EAIASLYVTK EGDTLDCROW ORVIALPGKL TMLSDOLTNV TVKRELYEIE

Ec 101 ROGNTIEYDG MTMERVDRPT AECAAALDKA PLPTPLP*

IIIII. IIII II. II III I III.I IIIIIII

St 101 RDGNTLEYDG MTLQRVARPT PEWPAALEKT PLPTPLPTPL $p *$

(b) Orf48 (92\% identity)

Ec 1 MSWQQFKHAW LIKFWAPIPA VIAAGILSTY YFGITGTFWA VTGEFTRWGG QLLQLFGVHA EEWGYFKIIH LEGSPLTRID GMMILGMFGG CFAAALWANN

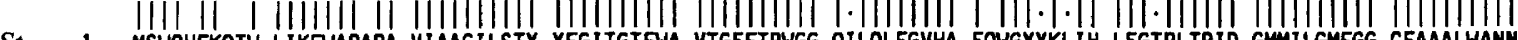
St 1 MSWQHFKQTH LIKFMAPAPA VIAAGILSTY YFGITGTFWA VTGEFTRWGG QILQLFGVHA EQWGYYKLIH LEGTPLTRID GWILGMFG CFAAALWANW

Ec 101 VKLRMPRSRI RIMQAIIGGI IAGFGARLAM GCNLAAFFTG IPQFSLHAWF FAIATAIGSW FGARFTLLPI FRIPVKMQKV SAASPLTQKP DQARRRFRLG St 101 VKLRMPRSRI RIVQAVAGGI \|\|$\|$ IAGFGARLAM GCNLAAFFTG IPQFSLHAWF FALATAIGSW FGARFTLLPI FRIPVKMQKV

Ec 201 MLVFFGMLGW ALLTAMNOPK LGLAMLFGVG FGLLIERAQI CFTSAFRDM ITGRTHMAKA IIIGMAVSAI GIFSYVQLGV EPKIMAGPN AVIGGLLFGF

St 201 MLVFIGMIGW ALLTAMHQPK LGLAMLFGVG FGLLIERAQI CFTSAFROLW ISGRAHAKA IIFGMAVSAI GIFSYVQLGV APKIMUAGPN AVIGGLLFGF

Ec 301 GIVLAGGCET GMMYRAVEGQ VHYWWGLGN VIGSTILAYY WDOFAPALAT DNOKINLLKT FGPMGGLLVT YLLLFAALML IIGWEKRFFR RAAPQTAKEI A*

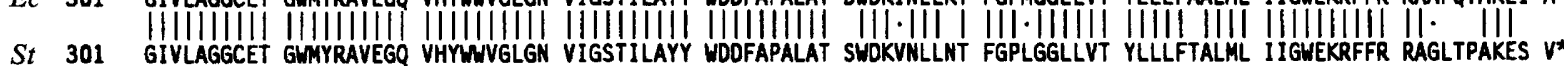

(c) Orf9 (100\% identity)

1 MKNIVPOYRL DMVGEPCPYP AVATLEAMPQ LKKGEILEVY SDCPQSINNI PLDARNHGYT VLDIQQOGPT IRYLIOK* MKNIVPDYRL DAVGEPCPYP AVATLEAMPQ LKKGEILEW SOCPQSINWI PLDARNHGYT VLDIQQDGPT IIIIIIQK*

1 MKNIVPDYRL DMVGEPCPYP AVATLEAMPQ LKKGEILEWV SOCPQSINNI PLDARNHGYT VLDIQQDGPT IRYLIQK*

Fig. 3. Comparison of the Orf15 $(a)$, Orf48 $(b)$ and Orf9 $(c)$ sequences of $E$. coli $(E c)$ and $S$. typhimurium (St). Identities are shown by vertical bars (l) and similarities (I/L/V/M/F, F/Y, A/G, D/E, H/K/R, N/Q, or S/T) by periods ( $)$.

third-base position are much more prevalent than mismatches at the other two positions, and are the major reason for the high amino acid similarity levels.

The conclusion from comparisons between $E$. coli and $S$. typhimurium, namely that these open reading frames correspond to real genes, is independently supported by evidence deriving from the individual sequences: (i) the open reading frames were scored as coding regions by the algorithm TESTCODE (Fickett, 1982) (see below) at confidence levels of $96,96,88,95,99$ and $96 \%$ for orf $15_{\mathrm{E}}$,

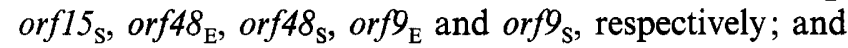
(ii) they all have strong, well-placed consensus ribosome binding sequences. orf $8_{\mathrm{E}}$ also appears to be an authentic gene by these criteria, and has a TESTCODE score of $96 \%$.

The Orf 15 , Orf48, Orf9 (and also Orf8 ${ }_{\mathrm{E}}$ ) sequences were compared against the GenBank database (version 75.0, February 1993) using TFASTA, but no significant similarities were found. In the absence of any clues to the functions of these proteins, we have not attempted to further identify and characterize them, but note briefly below some distinctive characteristics about their deduced amino acid sequences.

Orf15. This sequence has a basic N-terminus followed by a hydrophobic region which is, however, too short to be membrane-spanning. The C-terminus has the sequence TPLP repeated three times in the $S$. typhimurium sequence and (as APLPTPLP) twice in the E. coli sequence. $\mathrm{C}$-terminal repeats involving $\mathrm{T} / \mathrm{S}$ and $\mathrm{P}$ (but not of the precise form seen in Orf15) have been noted in a variety of DNA binding proteins, including RNA polymerase II and histones (Suzuki, 1990).

Orf48. This sequence is quite basic, with a total of $39 \mathrm{~K}+\mathrm{R}+\mathrm{H}$ residues compared with $17 \mathrm{D}+\mathrm{E}$ residues (E. coli data). The N-terminus is basic and is followed by the first of several (seven or eight) hydrophobic regions that are predicted to be membrane-spanning by the algorithm of Engelman et al. (1986). These extend throughout the entire sequence, and suggest that Orf48 is an integral membrane protein with no substantial aqueous domains.

Orf9. No special features were evident for this sequence, which appears to be that of a small soluble protein.

The apparently non-coding region immediately prior to $f i E$

As was described above, E. coli and $S$. typhimurium have 2.7 and $0.8 \mathrm{~kb}$ of DNA following a series of open reading frames and prior to the first gene of flagellar region IIIb, $f i E$. The absence of open reading frames of significant size (and also the complete lack of any sequence similarity between the two species) indicated that the DNA was non-coding. 
(a) E. coli

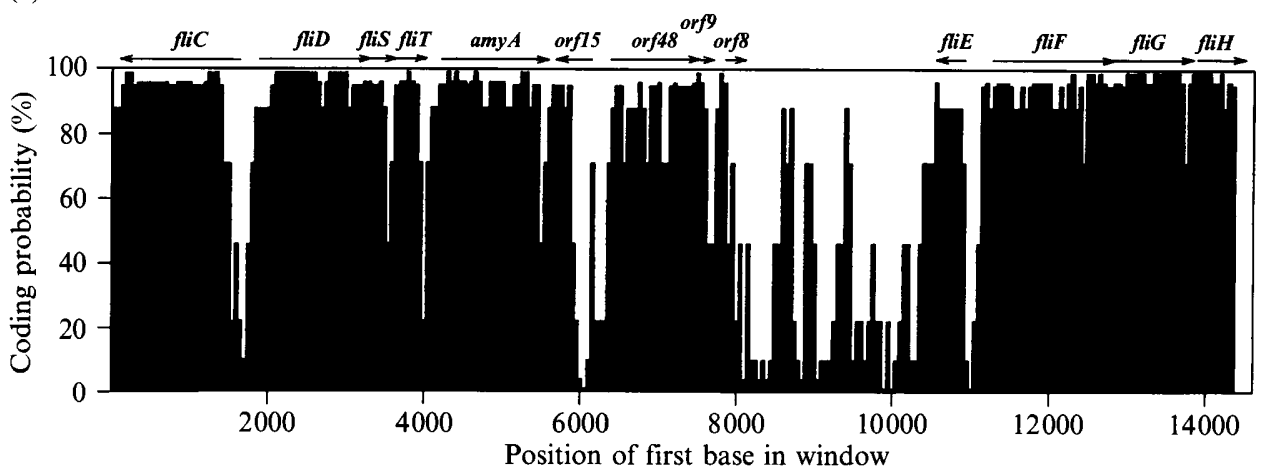

(a) S. typhimurium

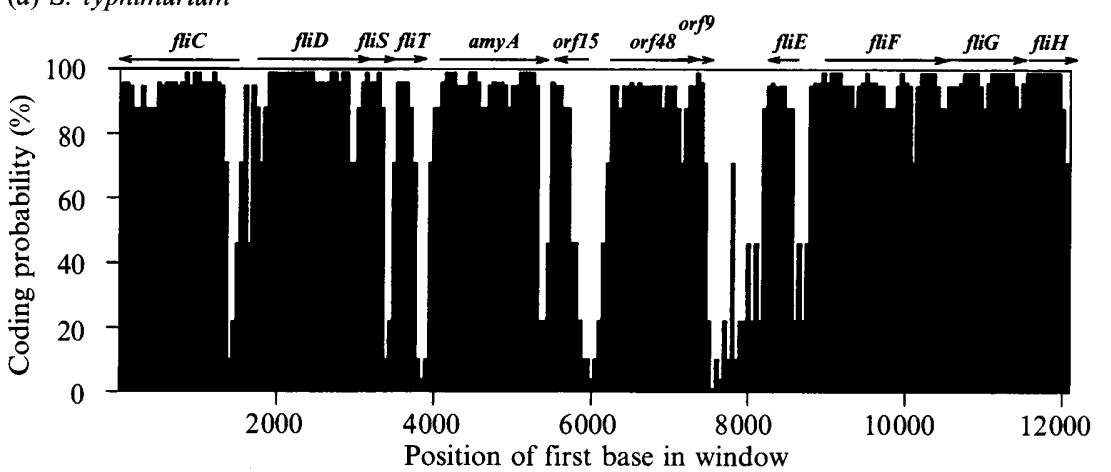

Fig. 4. Coding probability of the chromosomes of (a) E. coli and (b) S. typhimurium starting with the last four genes of flagellar region IIIa and continuing with the intervening region and the first four genes of flagellar region IIIb. The sequences were analysed by the TESTCODE algorithm of Fickett (1982), using a window of 200 residues and a step of 50 residues from one window to the next. As well as occasional random downward fluctuations in coding probability, there are sharp drops as the window passes through the non-coding region between operons and sometimes at the boundary between genes within an operon where there is a shift in frame. Note the extensive region of low coding probability between $\operatorname{orf} 8(E$. coli) or orf 9 (S. typhimurium) and $f i E$, and its much greater size in $E$. coli.

To test this further, we used the algorithm TESTCODE devised by Fickett (1982). This is based on autocorrelation analysis of nucleotides at step 3 , and scores the probability that DNA sequence is coding. No knowledge of frame is required, no attention is paid to stop codons, and the algorithm does not depend on signals such as ribosome binding consensus sequences. Typically (e.g. the open reading frames described above), authentic coding sequence scores as such with a confidence level of $90 \%$ or higher, whereas non-coding sequence scores very poorly. The algorithm can also be run using a scanning window: in this case, when an authentic reading frame is scanned the confidence level is typically in the 90th percentile most of the time with occasional downward fluctuations; in contrast, $5^{\prime}$ and $3^{\prime}$ non-coding sequences score as coding at around the 10-20th percentile confidence level, with occasional upward fluctuations.

When the $E$. coli DNA sequence spanning flagellar region IIIa, the intervening region, and flagellar region IIIb was examined in this way, the results were striking (Fig. $4 a$ ). All the way from the flagellar region IIIa genes through $\operatorname{orf}_{\mathrm{E}}$ (except for the non-coding regions between adjacent operons) coding probabilities were high; the same was true in flagellar region IIIb. Between $\operatorname{orf}_{\mathrm{E}}$ and $f i E_{\mathrm{E}}$, however, the coding probabilities were for the most part extremely low; there were a few spikes of higher probability, which might be statistical fluctuations or might represent local regions where coding information has not been entirely lost. A similar situation applied to $S$. typhimurium (Fig. $4 b$ ), but in this case the high probability region corresponding to $\operatorname{orf}_{\mathrm{E}}$ was lacking and the predicted non-coding region was much shorter, as the similarity matrix in Fig. 2 had already revealed.

Further evidence for absence of a coding function for this DNA came from base composition. Whereas coding sequence of DNA in $E$. coli and $S$. typhimurium commonly has an $\mathrm{A}+\mathrm{T}$ content of around $48 \%$, control and other non-coding regions generally have a higher $\mathrm{A}+\mathrm{T}$ content, often as high as $65 \%$ (Haughn et al., 1986). This was found to be true of the $2.7 \mathrm{~kb}$ region between $\operatorname{orf}_{\mathrm{E}}$ and $f i E_{\mathrm{E}}$ and the $0.8 \mathrm{~kb}$ region between orf $_{\mathrm{S}}$ and $f i E_{\mathrm{S}}$, where the mean $\mathrm{A}+\mathrm{T}$ content was $59 \%$ in both cases (data not shown). The later part of the $2.7 \mathrm{~kb}$ 
region in $E$. coli was especially AT-rich, with a mean content of $66 \%$ over more than $1.4 \mathrm{~kb}$.

In a search of the GenBank database using FASTA, we found no significant similarities to these non-coding sequences; the highest scoring examples (optimized scores in the range 100-150) were AT-rich regions from a variety of sequences.

The DNA in this region lacks any distinguishing characteristics that might provide a clue to its function. It contains some examples of inverted repeats scattered throughout the sequence, but probably not more than would be expected by chance; it does not contain any major direct repeats. No examples of the repetitive extragenic palindromic (REP) sequences that are scattered around the chromosomes of E. coli and $S$. typhimurium were found within these apparently noncoding regions; this result agrees with the physical mapping of REP sequences throughout the entire $E$. coli genome (Dimri et al., 1992), where the only one in the vicinity of flagellar regions IIIa and IIIb is REP40 at the $3^{\prime}$ end of $f i C_{\mathrm{E}}$ (Hanafusa et al., 1989).

\section{Discussion}

In this study, we have attempted to understand the organization and significance of the several kilobases of DNA that separates two major clusters of flagellar genes in both $E$. coli and S. typhimurium (Fig. $1 a, b$ ).

The fact that flagellar regions IIIa and IIIb are within only a few kilobases (i.e. about $0 \cdot 1 \%$ of the chromosome) of each other suggests to us that originally they were contiguous and then were subjected to rearrangement by insertion of DNA, either from elsewhere on the chromosome or from another source such as a plasmid. If this is true, the rearrangement must be an ancient one that occurred before the speciation of $E$. coli and $S$. typhimurium around 140 million years ago (Ochman \& Wilson, 1987). What is the nature of the inserted DNA, and what has happened to it in the time since it disrupted the ancestral flagellar region?

The intervening region was known to contain at least one gene, which codes for a cytoplasmic $\alpha$-amylase, AmyA (Raha et al., 1992). The analysis of the entire region in the present paper has revealed several other genes. Database comparisons provide no basis for suggesting that they play a role in sugar metabolism and so it appears that amy $A$ exists as an isolated gene remote from others that may have related roles, such as the $\mathrm{glg}$ (glycogen) cluster located at 76 min on the E. coli map (Bachmann, 1990).

Although the functions of orf 15 , orf 48 and orf9 are unknown, for several reasons we are confident that they are authentic genes. The most compelling argument is the very high level of amino acid identity for the deduced products in the two species. orf8, present only in $E$. coli, is probably an authentic gene also, although here we do not have the amino acid comparison to rely on.

The transition from the last flagellar region IIIa operon to amy $A$ and the other open reading frames described above is abrupt, with a typically short noncoding region. The same is true between amy $A$ and orf15, between orf15 and orf48, and (in E. coli) between orf 9 and orf8. From that point on, all the way to the boundary of flagellar region IIIb, the DNA sequence appears to be non-coding. There are four lines of evidence for a predominantly or exclusively non-coding function, which taken together are compelling: (i) the DNA contains no open reading frames of significant length; (ii) the DNA scores very poorly as coding sequence; (iii) the $\mathrm{A}+\mathrm{T}$ content is high, a characteristic of non-coding sequence; and (iv) there is no detectable similarity between the $E$. coli and $S$. typhimurium sequences. The existence of substantial amounts of noncoding DNA at the rightmost end of the intervening region is in striking contrast with the extremely tight apposition at the leftmost end, where apparently all redundant DNA between the region IIIa flagellar genes and amy $A$ has been squeezed out. Why the two ends of the intervening region should be so different is unclear.

We have examined the literature for other known examples of substantial regions of non-coding DNA on the $E$. coli or $S$. typhimurium chromosomes. Haughn et al. (1986) reported a $500 \mathrm{bp}$ gap between two divergent genes, leuP and an open reading frame of unknown function. More recently, as part of the ongoing E. coli genome project, Daniels et al. (1992) have described the sequence of $91 \mathrm{~kb}$ of DNA between 84.5 and $86.5 \mathrm{~min}$ on the chromosome; within that sequence they have found examples of large regions of apparently non-coding DNA, and have coined the phrase 'grey holes' for such regions. Interestingly, in another recent report of an extensive $(111 \mathrm{~kb})$ contiguous sequence of the $E$. coli chromosome, from 0 to $2.4 \mathrm{~min}$, no such grey holes were found (Yura et al., 1992). The largest grey hole described by Daniels et al. was $1.2 \mathrm{~kb}$. The one between flagellar regions IIIa and IIIb is, at $2.7 \mathrm{~kb}$, more than twice as large and is to our knowledge the largest that has been found thus far on the $E$. coli chromosome.

It is not known whether grey holes represent sequence that performs a useful function such as, for example, stabilizing higher-order chromosome structure. At least in the case of the grey hole between flagellar regions IIIa and IIIb, it is hard to imagine it having any such function, given its disparate sizes in $E$. coli and $S$. typhimurium.

If these extensive stretches of DNA are non-functional, how might they arise? Genetic rearrangements such as random insertions or deletions will, in general, cause 
disruption or loss of information at the rearrangement boundary, which statistically is likely to be within either a coding region or a control region. If this information is needed for expression of a number of genes (e.g. because it is the promoter of a multi-gene operon), or if loss of one gene product compromises the function of others (e.g. because these proteins form a multi-subunit complex or are components of a multi-enzyme pathway), several kilobases of DNA could easily be rendered useless by the rearrangement. At this point - unless the rearrangement was too deleterious to survive - the sequence would be free to drift.

However, while this scenario can explain the loss of coding content, and also the divergence between the $E$. coli and $S$. typhimurium sequences, it does not explain why the useless DNA has been retained. The implication is that the genetic load is small enough that it can be tolerated, much as phenotypically silent mutations are. But, if this is true, it is unclear why large non-coding regions are not commonplace in the bacterial chromosome.

In the case of S. typhimurium, at least, a substantial amount of this DNA has, in fact, been lost (at least twothirds, if we assume $E$. coli has lost little or none). It would be interesting in this regard to compare the DNA of a variety of wild-type $E$. coli strains to see whether this non-coding region differs in length and in sequence and, if so, by how much. It would also be interesting to examine this part of the chromosome in the genus that is most closely related to $E$. coli, namely, Shigella, which is non-motile but might still have remnants of the flagellar gene system.

Another question that arises is how such a major rearrangement could occur without damage to the flagellar gene system. It does not seem likely that in the ancestral genome there can have been any important flagellar genes between the $f i D$ and $f i E$ operons, since there are none now and yet motility has been retained. Yet if these operons were originally adjacent, the disruption must have occurred rather precisely within a non-essential part of sequence in order to leave them both intact. While this might seem a somewhat improbable event statistically, perhaps only such an event would have been able to survive in the face of the selection pressure in favour of motility. Interestingly, the genes in the two regions are now functionally somewhat different, with those in region IIIa being involved in the flagellar filament and other late components of the flagellar assembly process, while those in region IIIb are involved in earlier components such as the switch and the basal-body MS ring. Perhaps this differentiation is in some way related to the events that have caused the physical separation on the chromosome.

We thank Frederick Blattner and Kenneth Rudd for helpful discussions; David DeRosier, Nicholas Ornston and Sandy Parkinson for critically reading this manuscript; and David Morgan for carrying out the database comparisons. This work was supported by USPHS grant AI12202.

\section{References}

BACHMANN, B. J. (1990). Linkage map of Escherichia coli K-12, Edition 8. Microbiological Reviews 54, 130-197.

Daniels, D. L., Plunkett, G., III, Burland, V. \& Blattner, F. R. (1992). Analysis of the Escherichia coli genome: DNA sequence of the region from 84.5 to 86.5 minutes. Science 257, 771-778.

Dimri, G. P., Rudd, K. E., Morgan, M. K., Bayat, H. \& AmES, G. F.-L. (1992). Physical mapping of repetitive extragenic palindromic sequences in Escherichia coli and phylogenetic distribution among Escherichia coli and other enteric bacteria. Journal of Bacteriology 174, 4583-4593.

Engelman, D. M., Steitz, T. A. \& Goldman, A. (1986). Identifying nonpolar transbilayer helices in amino acid sequences of membrane proteins. Annual Review of Biophysics and Biophysical Chemistry 15, 321-353.

FICKETT, J. W. (1982). Recognition of protein coding regions in DNA sequences. Nucleic Acids Research 10, 5303-5318.

Hanafusa, T., Sakai, A., Tominaga, A. \& Enomoto, M. (1989). Isolation and characterization of Escherichia coli hag operator mutants whose hag48 expression has become repressible by a Salmonella H1 repressor. Molecular and General Genetics 216, 44-50.

Haughn, G. W., Wessler, S. R., Gemmill, R. M., Calvo, J. M. (1986). High A + T content conserved in DNA sequences upstream of leuABCD in Escherichia coli and Salmonella typhimurium. Journal of Bacteriology 166, 1113-1117.

Homma, M., DeRosier, D. J. \& MaCnab, R. M. (1990). Flagellar hook and hook-associated proteins of Salmonella typhimurium and their relationship to other axial components of the flagellum. Journal of Molecular Biology 213, 819-832.

Kawagishi, I., Müller, V., Williams, A. W., Irikura, V. M. \& MACNAB, R. M. (1992). Subdivision of flagellar region III of the Escherichia coli and Salmonella typhimurium chromosomes and identification of two additional flagellar genes. Journal of General Microbiology 138, 1051-1065.

Lipman, D. J. \& Pearson, W. R. (1985). Rapid and sensitive protein similarity searches. Science 227, 1435-1441.

Müller, V., Jones, C. J., KaWagishi, I., AizaWa, S.-I. \& Macnab, R. M. (1992). Characterization of the fiE genes of Escherichia coli and Salmonella typhimurium and identification of the FliE protein as a component of the flagellar hook-basal body complex. Journal of Bacteriology 174, 2298-2304.

OChMAN, H. \& Wilson, A. C. (1987). Evolutionary history of enteric bacteria. In Escherichia coli and Salmonella typhimurium. Cellular and Molecular Biology, vol. 2, pp. 1649-1654. Edited by F. C. Neidhardt, J. Ingraham, K. B. Low, B. Magasanik, M. Schaechter \& H. E. Umbarger. Washington, DC: American Society for Microbiology.

Raha, M., Kawagishi, I., Müller, V., Kihara, M. \& Macnab, R. M. (1992). Escherichia coli produces a cytoplasmic $\alpha$-amylase. AmyA. Journal of Bacteriology 174, 6644-6652.

SUZUKI, M. (1990). The heptad repeat in the largest subunit of RNA polymerase II binds by intercalating into DNA. Nature, London 344, 562-565.

Yura, T., Mori, H., Nagai, H., Nagata, T., Ishihama, A., Fujita, N., Isono, K., Mizobichi, K. \& NaKata, A. (1992). Systematic sequencing of the Escherichia coli genome: analysis of the 0-2.4 min region. Nucleic Acids Research 20, 3305-3308. 